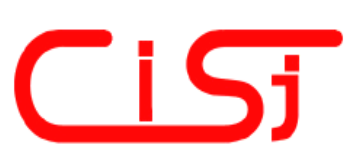

\title{
FUZZY RECURRENT MAPPINGS IN MULTIAGENT SIMULATION OF POPULATION DYNAMICS SYSTEMS
}

\author{
Dmytro Chumachenko 1), Oleksandr Sokolov ${ }^{2)}$, Sergiy Yakovlev 1) \\ 1) National Aerospace University "Kharkiv Aviation Institute", 17 Chkalov str., Kharkiv, 61070, Ukraine \\ dichumachenko@gmail.com, svsyak7@gmail.com \\ 2) Nicolaus Copernicus University in Torun, 11 Gagarin str., Torun, Poland, saszasokolov@ gmail.com
}

Paper history:

Received 27 February 2019

Received in revised form 26 July 2019

Accepted 17 February 2020

Available online 14 June 2020

\begin{abstract}
The article deals with the problems of analyzing multi-agent models of population dynamics. The problems studied are caused by a number of uncertainties associated with variables, boundary conditions, initial states, parameter values, etc. Given problems could be found in tasks associated with cyber security of critical infrastructures (e.g. DDoS attacks, computer worms, etc.). To solve this problem, a linguistic fuzzy model has been developed, which allows describing systems of population dynamics in a more realistic way. Population dynamics is described by a set of rules, each of which involves entry and exit in the form of fuzzy sets or fuzzy functions, which are applied iteratively. The complexity of describing the processes of population dynamics systems, the presence of fuzzification and defuzzification algorithms, and the use of fuzzy sets and linguistic variables make it necessary to develop new methods for analyzing such systems. The approaches proposed in the article to the study of systems of population dynamics make it possible to apply a unified description of processes of different nature in the form of a production set of rules.
\end{abstract}

Copyright $@$ Research Institute for Intelligent Computer Systems, 2020. All rights reserved.

\section{INTRODUCTION}

The dynamics of any system is described by a mathematical model that reflects the dependencies between input, output, and state variables [1]. The main property of any dynamic system is that its behavior at any time depends not only on variables acting on it at a given time, but also on variables acting on it in the past [2].

Multi-agent modeling is one of the most relevant ways to create simulation models [3]. When solving the problem of modeling dynamic systems, researchers are faced with the problem of describing the complex structure of interacting elements that are not homogeneous and cannot be typified by their properties [4]. Analytical models and traditional discrete event models are not effective, and the possibility of their use in certain systems is absent. In such cases, resort to the use of multi-agent modeling, built from the bottom up, i.e., global system dynamics is formed due to the interaction of autonomous models [5]. The rapidly increasing computing power of personal computers, which allows for the simulation of a large number of independent objects, is a significant catalyst for the development of multi-agent modeling as applied to population dynamics systems [6].

Researchers of population dynamics systems encounter a number of obstacles when trying to test their models, in particular, due to a number of uncertainties associated with variables, boundary conditions, initial states, parameter values, etc. [7]. In practice, only data on registered processes are available for research, in addition, such data demonstrate vagueness in the definition of such concepts as risk factors, hazards, exposure forces, contact patterns, etc. [8]. Thus, a possible alternative approach could be a combination of fuzzy logic methods and nonlinear dynamic systems in order to provide comprehensive analysis and development of prediction tools in population dynamics systems [9]. 
It is known that with the help of production models it is possible to naturally describe a person's declarative experience, his intuition and logic of behavior. It is also often advisable to use fuzzy linguistic variables, with which you can adequately reflect the approximate description of the interacting elements, in the case when the exact deterministic description is missing [10]. Also it can be useful in description of processes in cyber security of critical infrastructures, such as simulation of network worms [11] or DDoS attacks [12]. It should be noted that many fuzzy categories described linguistically are often no less informative than an exact description [13].

\section{PROBLEM STATEMENT}

The existing approaches have shown some theoretical difficulties, in connection with which, given the peculiarities of the mathematics of fuzzy logic, it is difficult to show effective practical results. In order to investigate how fuzzy logic can describe population dynamics systems in a more realistic way, this study has developed a linguistic fuzzy model applied to the multi-agent model described in [14].

\section{STUDY OF THE STABILITY OF THE MODEL OF POPULATION DYNAMICS}

An effective model provides accurate results with small deviations over a long period. Input $x$, output $y$, and state must be present in any system modeling dynamic processes [15]. Many models provide high results, but only for a short time. Others succeed only in the presence of a very limited set of initial assumptions, which may be a pure coincidence of circumstances [16]. A model that deserves serious attention must be sustainable in the sense that it provides equally good results under different conditions and for different periods [17].

Let us consider the population of a dynamic system. It is necessary to find out how the number of $N_{k+1}$ of $(k+1)$-th year individuals is related to the number of $N_{k}$ of $k$-th individuals. In the simplest case, we can assume that

$$
N_{k+1}=A \cdot N_{k},
$$

where $A$ is a number, depends on environmental conditions.

If $A>1$, then the population will grow; if $A<1$, the number will decrease

Let us consider limitations that are more realistic. Population growth is limited. We can include this limiting feature by adding another model variable, which will be negligibly small for small $N$ values, but becomes more important with increasing $N$. One possible way to do this is to enter a term proportional to $N^{2}$, which will lead to

$$
N_{l}=A \cdot N_{0}-B \cdot N_{0}^{2}
$$

If $B$ is significantly less than $A$, then the second term in (2) will not matter until $N$ is large enough. The minus sign means that the second member tends to decrease in population. In the next step, we use equation (2) to find out how $N$ changes in subsequent years.

$$
\begin{gathered}
N_{2}=A \cdot N_{l}-B \cdot N_{1}^{2}, \\
N_{3}=A \cdot N_{2}-B \cdot N_{2}{ }^{2}, \\
\quad \ldots \\
N_{k+1}=A \cdot N_{k}-B \cdot N_{k}{ }^{2} .
\end{gathered}
$$

In this case, the maximum possible number of population is

$$
N^{\max }=A / B
$$

We introduce the concept of the proportion of the maximum possible population

$$
x_{k}=N_{k} / N^{\max } .
$$

Substituting (5) into (1), we get

$$
x_{n+1}=A x_{n}\left(1-x_{n}\right)=f_{A}\left(x_{n}\right),
$$

where $\mathrm{x}_{\mathrm{n}}$ is the population in the $n$-th year.

The next step is to test the intelligent multi-agent model of epidemic processes for sustainability. To do this, we study the hepatitis B incidence model set up in paper [18]. Since the model is not linear and is not represented by mathematical dependencies, and the model output is the result of the interaction of intelligent agents with each other and with the external environment, it is natural to test the system for stability changes in output $\Delta y$ from changes in input parameters $\Delta x$. As a result of the conducted experiments, insignificant differences were revealed as a result of the model, namely

$$
\mathrm{f}(\Delta x)=\Delta y=0,0057 \text {. }
$$

\section{CURRENT STATE OF RESEARCHES}

Researchers of dynamic systems encounter a number of obstacles when trying to test their models, 
in particular, due to uncertainties associated with variables, boundary conditions, initial states and parameter values, etc. Ideally, experts should take them from actual data on a dynamic system and the analysis of this data, but in reality, only data on registered processes are available for research, moreover, such data demonstrate vagueness in defining such concepts as risk factors, danger, impact strength, contact patterns or infection status [19]. Thus, a possible alternative approach may be a combination of fuzzy logic methods and nonlinear dynamic systems in order to provide comprehensive analysis and development of prediction tools in dynamic systems with an epidemic character.

Fuzzy dynamic systems are relatively new field of research, the main idea of which is to expand the standard dynamic system modeled using differential equations or another approach to the theoretical framework of a fuzzy set. These methods allow one to take into account the uncertainties associated with variables, parameters, boundary conditions and initial states and to model their evolution, adhering to the basic rules and regularities of the system dynamics.

Fuzzy rule-based models are systems whose variables are described by fuzzy sets, not clear numbers. They are based on the concept of fuzzy information breaking and can be classified into two general groups depending on how the information is presented: 1) linguistic models, in which the most famous example is the Mamdani type model, and 2) the Takagi - Sugeno model. Both models are based on the use of fuzzy rules and linguistic variables. However, linguistic models are a qualitative description of the behavior of a system using natural language, and the Takagi-Sugeno model is a combination of fuzzy and standard structures.

Successful use of a fuzzy linguistic model in modeling controllers shows that this is the most applied structure. The fuzzy linguistic model is based on approximate reasoning, which provides the basis for building hypotheses with inaccurate information with the help of adequate inference mechanisms [20]. This model can be defined as an expert system, because it has a knowledge base and an inference mechanism, both of which are based on human expert knowledge. Most fuzzy applications are based on fuzzy linguistic systems. They are widely used in the development of fuzzy controllers of medical devices, risk assessment and diagnostic systems [21-24]. There are also several fuzzy linguistic systems in studies of epidemic processes $[25,26]$.
Fuzzy rule-based models have a simple structure and consist of four main components: 1) the fuzzification module, which translates clear inputs (classical measurements) into fuzzy values using linguistic variables 2) an indistinct base of rules IfThen, which consists of a set conditional fuzzy judgments; 3) an inference method that uses fuzzy mechanisms to obtain results or, in other words, a calculation method with fuzzy rules; 4) the defuzzification module, which translates fuzzy outputs back to clear values, if necessary. A typical model of a fuzzy rule-based model and the relationship of its modules are shown in Fig. 1.

In [27], a structure was proposed for solving systems of linear differential equations for one class of fuzzy sets, based on the $\alpha$-level. However, the proposed methods are difficult to apply in dynamic systems with an epidemic nature, since such models have obvious non-linearities and should be considered differently. These non-linearities are due to the fact that the course of the epidemic process of a dynamic system depends, among other things, on the proportion of those in various states that are by their nature uncertain and, therefore, are ideal objects for analysis using fuzzy logic.

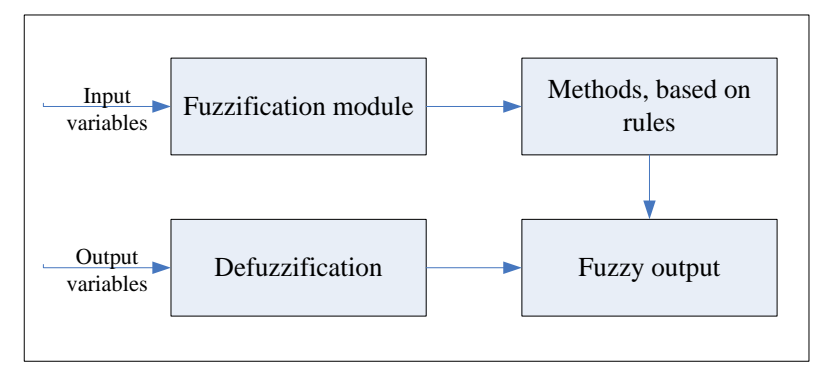

Figure1 - Diagram of a typical fuzzy rule-based model

Papers [28, 29] have proposed a new approach to the description of the dynamics of the ecological model in differential equations, which reproduces the dynamic system using fuzzy parameters. In this case, the solution to the system of equations is the so-called fuzzy expected value. The application of this approach in epidemiological systems is not obvious because some details and parameters can be interpreted ambiguously. Despite this, the method is a possible way to model the epidemic system more realistically.

Both approaches had some theoretical difficulties, in connection with which, given the peculiarities of the mathematics of fuzzy logic, it is difficult to show effective practical results. In order to investigate how fuzzy logic can describe the dynamics of epidemiological systems in a more 
realistic way, this research examines a linguistic fuzzy model applied to the multiagent model described in paper [18].

\section{APPLICATION OF FUZZY RULES TO THE DESCRIPTION OF POPULATION DYNAMICS}

The main idea of this approach is that the system dynamics is described by a set of rules that are applied iteratively. Each rule provides input and output in the form of fuzzy sets or fuzzy functions. From the empirical experience of the group of experts it is possible to create a fuzzy membership function for each variable and / or parameter, as well as linguistic rules governing the dynamics of the system. Thus, a fuzzy model consists of a set of rules and the corresponding inference of a finite state machine. The linguistic model takes the following form:

$$
\begin{aligned}
& \operatorname{IF} U=B_{1} \text { AND } W_{1}=A_{11} \text { AND } \ldots \text { AND } W_{n}=A_{1 n} \\
& \operatorname{THEN} \bar{W}_{1}=\hat{A}_{11} \text { AND } \ldots \text { AND } \bar{W}_{n}=\hat{A}_{1 n} \text { AND } V=D_{1} \\
& \operatorname{IF} U=B_{2} \text { AND } W_{1}=A_{21} \text { AND } \ldots \text { AND } W_{n}=A_{2 n} \\
& \operatorname{THEN} \bar{W}_{1}=\hat{A}_{21} \text { AND } \ldots \text { AND } \bar{W}_{n}=\hat{A}_{2 n} \text { AND } V=D_{2}
\end{aligned}
$$

$$
\begin{gathered}
\operatorname{IF} U=B_{m} \text { AND } W_{1}=A_{m 1} \text { AND } \ldots \text { AND } W_{n}=A_{m n} \\
\operatorname{THEN} \bar{W}_{1}=\hat{A}_{m 1} \text { AND } \ldots \text { AND } \bar{W}_{n}=\hat{A}_{m n} \text { AND } V=D_{m},
\end{gathered}
$$

where $U$ is the input variable, $W_{i}$ is the system state variable, $V$ is the output variable, $\bar{W}_{i}$ is the system state variable after each iteration, $B_{i}$ and $A_{i j}$ are the input fuzzy sets, $D_{i}$ and $\hat{A}_{i j}$ are the output fuzzy sets.

In this regard, choosing the appropriate inference method and, if necessary, the defuzzification method, at each step after launching the model, the value of the state variable is calculated, which will be the input parameter of the system in the next step, and so on, iteratively.

This implies the following:

$$
\begin{aligned}
U(l+1) & =V(l), \\
W_{i}(l+1) & =\bar{W}_{i}(l),
\end{aligned}
$$

where $(l+1)$ is the next step after 1 .

There are other fuzzy dynamic systems, and the choice of a particular model depends on the type of information available about the system. Sometimes a part of the system's rules of behavior is known in advance, and then the rules will take the following form

$$
\begin{aligned}
& \operatorname{IF} U(l)=B_{m} \text { AND } W_{1}(l)=A_{m 1} \text { AND } \ldots \text { AND } \\
& W_{n}(l)=A_{m n} \\
& \text { THEN } y(l+1)=f\left(U(l), W_{1}(l), \ldots, W_{1}(l)\right) ;
\end{aligned}
$$

where $y(l+1)$ is some a priori function known from system dynamics.

Developing fuzzy models with experts in various fields requires interdisciplinary relationships [30]. For the correct application of expert knowledge, it is important that they build fuzzy sets. In general, the fuzzy sets of epidemiological systems built by experts do not show behavior. They tend to asymmetric and irregular dynamics, different from the behavior of fuzzy sets in engineering areas. In addition, experts have problems with understanding the nature of system dynamics, rules of behavior and, as a result, the model as a whole [31]. In addition, the creation of consequences from the rules of behavior is a more time-consuming task for an expert than analyzing past statistics of a dynamic system, because the expert must study the issue of system dynamics, taking into account all factors and formulation of a specific relationship, corresponds to the membership function. In addition, in order to analyze past statistics, which can influence the further behavior of a dynamic system, it is sufficient for an expert to classify the variables of the membership function. Therefore, in general, the expert has more opportunities to develop the prerequisites for the development of the epidemic process than the consequences.

Given these characteristics, the method of developing a posteriori linguistic rules can mean important progress in modeling dynamic systems that have a high level of uncertainty, inaccuracy or ambiguity in the definition of variables and parameters. In addition, it is necessary to take into account that epidemic systems are dynamic, nonautonomous, and open and, therefore, have the capacity for input-output of sufficiently large amounts of data for constructing hybrid models extremely rarely.

Thus, it is possible to formalize a fuzzy recurrent mapping [32], which is determined by the set of rules $\left(r_{1}, r_{2}, \ldots, r_{N}\right)$, linking the states $\left(x_{1}^{t}, x_{2}^{t}, \ldots, x_{N}^{t}\right)$ and $\left(x_{1}^{\tau}, x_{2}^{\tau}, \ldots, x_{N}^{\tau}\right)$ of the 
dynamic system at present $\tau$ and future t points of time respectively:

$$
\left(x_{1}^{t}, x_{2}^{t}, \ldots, x_{N}^{t}\right)=\left(\begin{array}{c}
r_{1} \\
r_{2} \\
\ldots \\
r_{N}
\end{array}\right)\left(\begin{array}{c}
x_{1}^{\tau} \\
x_{2}^{\tau} \\
\ldots \\
x_{N}^{\tau}
\end{array}\right)^{T}
$$

Sets of rules (12) are

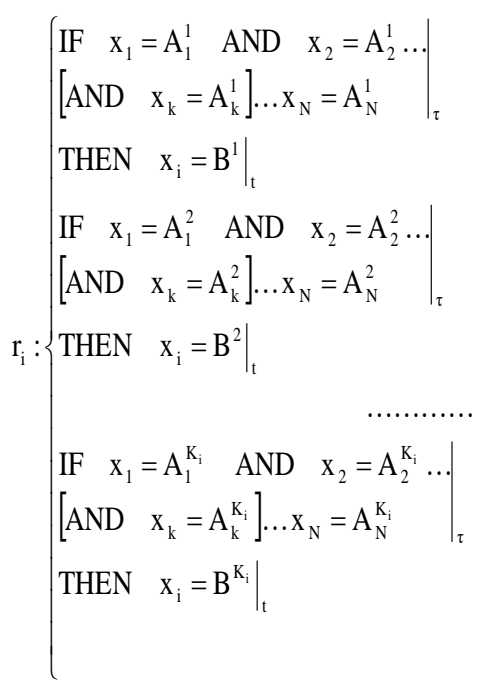

where $K_{i}$ is the number of rules in the set $r_{i}$; elements in square brackets are optional, that is, not all state variables can be involved in the rule; $A_{i}, B-$ the value of linguistic variables from the corresponding term-sets.

It is easy to show that the number of set rules is in the range

$$
0 \leq K_{i} \leq \prod_{\mathrm{i}=1}^{\mathrm{N}} \operatorname{card}\left(\mathrm{S}\left(\mathrm{x}_{\mathrm{i}}\right)\right)
$$

where card $\left(S\left(x_{i}\right)\right)$ is the power of a term-set of the linguistic variable $x_{i}$. Empty rule sets are also allowed.

The analysis of dynamic linguistic systems, presented in the general form (12), (13), is rather difficult, despite the high dimensions of the state space and the non-linear implicative nature of the relationship between events that transform the system from one state to another. In this regard, it is important to find such a form of describing the dynamics of the process, which would allow solving the problems of analysis and synthesis by formal methods. Usually in practice linguistic description in the form of rules are used.

$$
\begin{aligned}
& \text { IF } X_{k}=\left(x_{l=n} b, x_{2}=p m, \ldots, x_{n=} e\right) \\
& \text { AND } U_{k}=\left(u_{1}=p m, u_{2}=n b, \ldots, u_{m}=n m\right),(15) \\
& \text { THEN } X_{k+l}=\left(x_{l}=p b, x_{2}=p s, \ldots, x_{n=} p b\right),
\end{aligned}
$$

reflecting the ratio of changes in the state of the system depending on the input actions

$$
X_{k+1}=X_{k}{ }^{\circ} U_{k},
$$

where $X_{k}=\left(x_{1}, x_{2}, \ldots, x_{n}\right)_{k}$ is the generalized state vector of the system, and $U_{k}=\left(u_{1}, u_{2}, \ldots, u_{m}\right)_{k}$ is the generalized vector of control actions whose values are linguistic variables with the given term sets $S=\{n b, n m, \ldots, z e, \ldots, p m, p b\}$, where $n b-n e g a t i v e$ big, $\mathrm{nm}$ - negative middle, ze - zero, pm - positive middle, $p b$ - positive big are fuzzy sets with given membership functions [33].

The relation (16) can be represented as a network of transitions (Fig. 2) of generalized linguistic states (graph vertices) under the action of generalized linguistic control actions (graph edges). If $N$ is the dimension of the state vector $X, P$ is the dimension of the control vector $U, M$ is the power of a term-set of linguistic variables $S$, then the maximum possible number of vertices of the network (system states) is $M \cdot N$, and the number of arcs connecting these vertices (control actions ), $-M \cdot N \cdot((M N-1) / 2) \cdot M P$.

The analysis of such systems based on simulation modeling and the synthesis of optimal rules (the value of network edges) constitute a combinatorial problem.

In practice, instead of the general form of mapping (16), its individual forms are used when the vectors $X, Y, U$ are scalar linguistic variables. In the case when the consequent rules are linguistic, the model under consideration is the Mamdani model [34], and if functional, it is the Sugeno model [35].

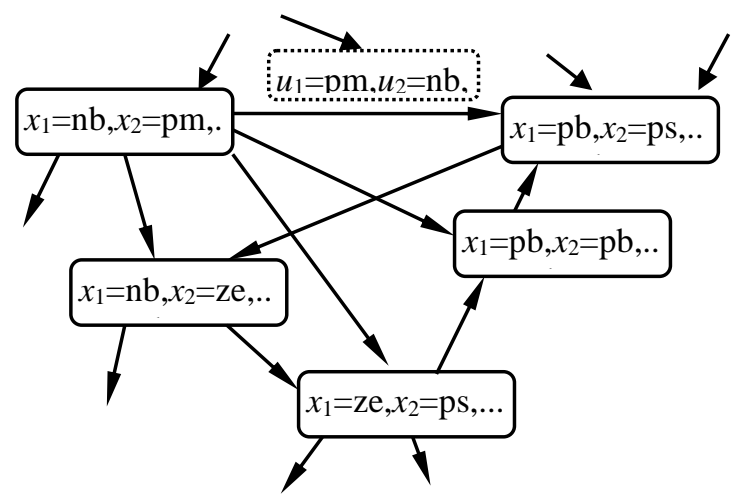

Figure 2 - Transition graph of a recurrent fuzzy system 
As a rule, the dynamic behavior of such systems is described in the form of tables of linguistic rules relating the control actions $U$ and the outputs (or states) of the object $X$. An example of such a display is presented in Table 1 .

The main problem of analyzing the model (16) is the absence of formal methods in the space of linguistic states similar to numerical models and methods of analysis and synthesis in the Euclidean state space, which makes it difficult to solve problems of analyzing the stability of a dynamic model, synthesizing optimal control systems and other problems. Known methods for analyzing fuzzy dynamic systems are based either on the study of membership functions of fuzzy sets, or on the analysis of transitions in the space of states, extended, or on heuristic methods of linguistic dynamics [36]. In addition, the dimensionality of the set of linguistic rules is significant, in which the number of possible rules increases exponentially with the number of input variables.

Table 1. Linguistic rules $X_{k+1}=X_{k} \boldsymbol{U}_{k}$

\begin{tabular}{|l|l|l|l|l|l|}
\hline $\mathbf{U}_{\mathbf{k}} \mathbf{X}_{\mathbf{k}}$ & $\mathbf{n b}$ & $\mathbf{n m}$ & $\mathbf{z e}$ & $\mathbf{p m}$ & $\mathbf{P b}$ \\
\hline $\mathbf{n b}$ & $\mathrm{nb}$ & $\mathrm{nb}$ & $\mathrm{nb}$ & $\mathrm{nm}$ & $\mathrm{Ze}$ \\
\hline $\mathbf{n m}$ & $\mathrm{nb}$ & $\mathrm{nb}$ & $\mathrm{nm}$ & $\mathrm{ze}$ & $\mathrm{Pm}$ \\
\hline ze & $\mathrm{nb}$ & $\mathrm{nb}$ & $\mathrm{ze}$ & $\mathrm{pb}$ & $\mathrm{Pb}$ \\
\hline $\mathbf{p m}$ & $\mathrm{nm}$ & $\mathrm{ze}$ & $\mathrm{pm}$ & $\mathrm{pb}$ & $\mathrm{Pb}$ \\
\hline $\mathbf{p b}$ & $\mathrm{ze}$ & $\mathrm{pm}$ & $\mathrm{pb}$ & $\mathrm{pb}$ & $\mathrm{Pb}$ \\
\hline
\end{tabular}

The complexity of describing the epidemic processes of dynamic systems, the presence of heuristic algorithms for fuzzification and defuzzification, as well as the use of fuzzy sets and linguistic variables lead to the need to develop new methods for analyzing such systems.

\section{CONCLUSIONS}

In given research dependences in the dynamics of the behavior of systems of population dynamics, the maximum possible number of individuals of a population depending on the input data and the relationship of future and current states of the system are formalized. The developed intellectual multiagent system of epidemic processes in population dynamics systems was tested for stability by the example of setting up a model for predicting the incidence of viral hepatitis B.

A number of obstacles are considered in checking simulation models for accuracy and adequacy, as well as in attracting experts from the field of the simulated system. It is shown that applying models based on the use of fuzzy linguistic rules can be a case of solving those problems.

A model of the epidemic process based on the application of fuzzy linguistic rules has been built. These methods allow one to take into account the uncertainties associated with variables, parameters, boundary conditions and initial states and to model their evolution, adhering to the basic rules and regularities of the system dynamics. Given model could be used not only for Public Health issues, but also for simulation epidemic processes in critical infrastructures, such as network work propagation or DDoS attacks simulation.

The next step in research will be estimation of the duration of the forecast model in terms of chaotic dynamics.

\section{REFERENCES}

[1] N. Gilbert, Analyzing Tabular Data: Loglinear and Logistic Models for Social Researchers, London: UCL Press, 1993, pp. 10-14.

[2] B. Chaib-draa, F. Dignum, "Trends in agent communication language," Computational Intelligence, vol. 18, issue 2, pp. 89-101, 2002.

[3] Y. Shoham, K. Leyton-Brown, Multiagent Systems - Algorithmic, Game-Theoretic, and Logical Foundations, Cambridge University Press, 2009, 483 p.

[4] J. Hollan, E. Hutchins, D. Kirsh, "Distributed cognition: toward a new foundation for humancomputer interaction research," $A C M$ Transactions on Computer-Human Interaction (TOCHI), vol. 7, issue 2, pp. 174-196, 2000.

[5] P. Maes, "Agents that reduce work and information overload," Communications of the ACM, vol. 37, pp. 31-40, 1994.

[6] T. Rahwan, T. Michalak, M. Wooldridge, N. R. Jennings, "Towards anytime coalition structure generation in multi-agent systems with positive or negative externalities," Artificial Intelligence, vol. 186, pp.95-122, 2002.

[7] M. J. Osborne, A. Rubinstein, A Course in Game Theory, Massachusetts, Cambridge: The MIT Press, 2014, 352 p.

[8] C. H. Papadimitriou, J. N. Tsitsiklis, "The complexity of Markov decision processes," Mathematics of Operations Research, vol. 12, issue 3, pp. 441-450, 1987.

[9] R. Conte, R. Hegselmann, P. Terna, "Simulating social phenomena," Lecture Notes 
in Economics and Mathematical Systems, vol. 456, 1997, $536 \mathrm{p}$.

[10] F. S. Santos, N. R. S. Ortega, D. M. T. Zanetta, E. Massad, "Fuzzy Dynamical model of epidemic spreading taking into account the uncertainties in individual infectivity," Advances in Technological Applications of Logical and Intelligent Systems, IOS Press, pp. 180-193, 2009.

[11] D. Chumachenko, S. Yakovlev, "On Intelligent Agent-Based Simulation of Network Worms Propagation," Proceedings of the 2019 15th International Conference on the Experience of Designing and Application of CAD Systems (CADSM), 2019, pp. 3.11-3.13.

[12] B. Zhang, T. Zhang, Z. Yu, "DDoS detection and prevention based on artificial intelligence techniques," Proceedings of the 2017 3rd IEEE International Conference on Computer and Communications (ICCC), Chengdu, 2017, pp. 1276-1280.

[13] D. Chumachenko,

V. Balitskii, T. Chumachenko, V. Makarova, M. Railian, "Intelligent expert system of knowledge examination of medical staff regarding infections associated with the provision of medical care," CEUR Workshop Proceedings, vol. 2386, 2019, pp. 321-330.

[14] I. Meniailov, K. Bazilevych, K. Fedulov, S. Goranina, D. Chumachenko, "Using the Kmeans method for diagnosing cancer stage using the Pandas library," CEUR Workshop Proceedings, vol. 2386, 2019, pp. 107-116.

[15] R. S. Sutton, A. G. Barto, Reinforcement Learning: An Introduction (Adaptive Computation and Machine Learning), The MIT Press, 2017, 322 p.

[16] N. Gilbert, K. G. Troitzsch, Simulation for the Social Scientist, Open University Press, 2005, pp. 172-199.

[17] L. Steels, "The Artificial Life Roots of Artificial Intelligence", Artificial Life: an overview, the MIT Press, 2000, pp. 75-110.

[18] D. Chumachenko, "On intelligent multiagent approach to viral hepatitis B epidemic processes simulation," Proceedings of the 2018 IEEE 2nd International Conference on Data Stream Mining and Processing, DSMP'2018, 2018, pp. 415-419.

[19] T.-Y. Li, J. A. Yorke, "Period three implies chaos," Amer. Math. Monthly, vol. 82, no. 10, pp. 985-992, 1975.
[20] R. Kempf, J. Adamy, "Regularity and chaos in recurrent fuzzy systems," Fuzzy Sets and Systems, vol. 140, issue 2, pp. 259-284, 2003.

[21] E. Massad, N.R.S. Ortega, L.C. de Barros, C.J. Struchiner, "Fuzzy dynamical systems in epidemic modeling," in: Fuzzy Logic in Action: Applications in Epidemiology and Beyond. Studies in Fuzziness and Soft Computing, vol 232. Springer, Berlin, Heidelberg, 2008, pp. 181-206.

[22] R. R. Yager, D. P. Filev, Essentials of Fuzzy Modeling and Control, N.Y.: Wiley, 1994, $408 \mathrm{p}$.

[23] M. Mahfouf, A. J. Asbury, D. A. Linkens "Physiological modelling and fuzzy control of anaesthesia via vaporisation of isoflurane by liquid infusion," International Journal of Simulation Systems, Science and Technology, vol. 2, issue 1, pp. 55-66, 2001.

[24] M. A. R. B. Castanho, Membrane-Active Peptides: Methods and Results on Structure and Function, International University Line, 2010, $635 \mathrm{p}$.

[25] A. P. Duarte, J. C. Bordado, M. T. Cidade, "Cellulose acetate reverse osmosis membranes: Optimization of preparation parameters," Journal of Applied Polymer Science, vol. 103, issue 1, pp. 134-139, 2006.

[26] H. Tanaka, N. R. S. Ortega, M. S. Galizia, J. B. Borges, M. B. P. Amato, "Fuzzy modeling of electrical impedance tomography images of the lungs," Clinics, vol. 63, issue 3, pp. 363-370, 2008.

[27] L. F. C. Nascimento, N. R. S. Ortega, "Fuzzy linguistic model for evaluating the risk of neonatal death," Revista de Saúde Pública, vol. 36, no. 6, pp. 686-692, 2002.

[28] R. M. Jafelice, L. C. de Barros, R. C. Bassanezi, F. Gomide, "Fuzzy modeling in symptomatic HIV virus infected population," Bulletin of Mathematical Biology, vol. 66, issue 6, pp. 1597-1620, 2004.

[29] L. C. Barros, R. C. Bassanezi, W. A. Lodwick, A First Course in Fuzzy Logic, Fuzzy Dynamical Systems, and Biomathematics, Springer-Verlag Berlin Heidelberg, 2017, 297 p.

[30] V.P. Mashtalir, S.V. Yakovlev, "Point-set methods of clusterization of standard information," Cybernetics and Systems Analysis, vol. 37, no. 3, pp. 295-307, 2001.

[31] L. C. Barros, M. B. F. Leite, R. C. Bassanezi "The SI epidemiological models with a fuzzy 
transmission parameter," International Journal of Computational Mathematical Applications, vol. 45, pp. 1619-1628, 2003.

[32] N. Ortega, L. C. Barros, E. Massad, "Fuzzy gradual rules in epidemiology," Kybernetes, vol. 32, issue 3, pp. 460-477, 2015.

[33] N. R. S. Ortega, P. C. Sallum, E. Massad, "Fuzzy dynamical systems in epidemic modeling," Kybernetes, vol. 29, issue 2, pp. 201-218, 2000.

[34] Z. Weihong, X. Shunqing, M. Ting, "A fuzzy classifier based on Mamdani fuzzy logic system and genetic algorithm," Proceedings of the 2010 IEEE Youth Conference on Information, Computing and Telecommunications, Beijing, 2010, pp. 198201.

[35] R. Kempf, J. Adamy, "Regularity and chaos in recurrent fuzzy systems," Fuzzy Sets and Systems, vol. 140, issue 2, pp. 259-284, 2003.

[36] R. S. Bernstein, "Multi-level simulation analysis: A methodology for planning and evaluation in public health," The many faces of multi-level issues (Research in Multi Level Issues), vol. 1, pp. 381-386, 2002.

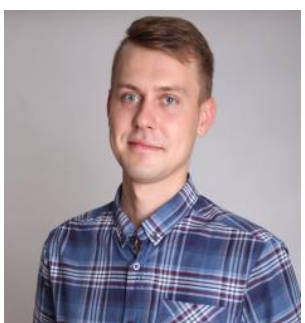

Dmytro Chumachenko, is $P h D$ in Artificial Intelligence, associate professor of the department of Mathematical modelling and artificial intelligence at National Aerospace University "Kharkiv Aviation Institute". Master's degrees in Social In-

formatics and Project Management. Scientific and professional interests are simulation, fuzzy logic, simulation of epidemic processes, agent based simulation, systems and means of Artificial Intelligence.

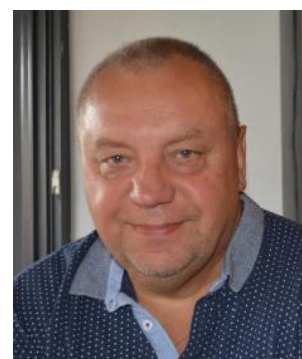

Oleksandr Sokolov, Prof. $d r$ hab. inż., Department of Informatics, Faculty of Physics, Astronomy and Informatics, Nicolaus Copernicus University, Torun, Poland.

Research Fields: Fuzzy Logic, Desicion Support Systems, Intelligent Control Sys-

tems, Medical Data Analysis.

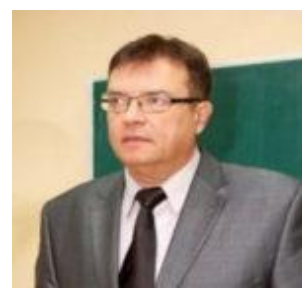

Sergiy Yakovlev, Prof., Dr. Sc. (Math.), professor of the Department of Mathematical modelling and artificial intelligence at National Aerospace University "Kharkiv Aviation Institute".

Professional interests are mathematical modelling, operation research, combinatorial optimization, global optimization, computational intelligence geometrical design. 